\title{
Mechanical Properties and Thermal Stability of TiN/Ta Multilayer Film Deposited by Ion Beam Assisted Deposition
}

\author{
Hongfei Shang, ${ }^{1,2}$ Jian $\mathrm{Li}^{1}{ }^{1}$ and Tianmin $\mathrm{Shao}^{2}$ \\ ${ }^{1}$ Wuhan Research Institute of Materials Protection, Wuhan 430030, China \\ ${ }^{2}$ State Key Laboratory of Tribology, Tsinghua University, Beijing 100084, China \\ Correspondence should be addressed to Tianmin Shao; shaotm@tsinghua.edu.cn
}

Received 1 May 2013; Accepted 24 October 2013; Published 28 January 2014

Academic Editor: Aiguo Xu

Copyright (c) 2014 Hongfei Shang et al. This is an open access article distributed under the Creative Commons Attribution License, which permits unrestricted use, distribution, and reproduction in any medium, provided the original work is properly cited.

\begin{abstract}
TiN/Ta multilayer film with a modulation period of $5.6 \mathrm{~nm}$ and modulation ratio of $1: 1$ was produced by ion beam assisted deposition. Microstructure of the as-deposited TiN/Ta multilayer film was observed by transmission electron microscopy and mechanical properties were investigated. Residual stress in the TiN/Ta multilayer film was about $72 \%$ of that of a TiN monolayer film with equivalent thickness deposited under the same conditions. Partial residual stress was released in the Ta sublayers during deposition, which led to the decrease of the residual stress of the TiN/Ta multilayer film. Nanohardness $(H)$ of the TiN/Ta multilayer film was $24 \mathrm{GPa}, 14 \%$ higher than that of the TiN monolayer film. It is suggested that the increase of the nanohardness is due to the introduction of the Ta layers which restrained the growth of TiN crystal and led to the decrease of the grain size. A significant increase (3.5 times) of the $H^{3} / E^{2}$ (E elastic modulus) value indicated that the TiN/Ta multilayer film has higher elasticity than the TiN monolayer film. The $L_{c}$ (critical load in nano-scratch test) value of the TiN monolayer film was $45 \mathrm{mN}$, which was far lower than that of the TiN/Ta multilayer film (around $75 \mathrm{mN}$ ). Results of the indentation test showed a higher fracture toughness of the TiN/Ta multilayer film than that of the TiN monolayer film. Results of differential scanning calorimetric (DSC) and thermo gravimetric analysis (TGA) indicate that the TiN/Ta multilayer film has better thermal stability than the TiN monolayer film.
\end{abstract}

\section{Introduction}

TiN thin film has been widely used for bearings, gears, and cutting tools due to its high hardness, good wear resistance, and anticorrosion performance. However, due to the relatively large residual stress and weak fracture toughness, TiN film cannot meet the more stringent requirements in many cases. Multilayer films have attracted great attention in recent years [1] because of their promising properties [2]. Much of the work has been focused on multilayer films based on TiN [3-16]. In recent years, TiN/metal multilayer films such as TiN/Ti [11-14], TiN/Cu [15], and TiN/W [16] have been extensively studied. Multilayer films of nitride/metal may have a higher hardness than the nitride itself, although the metal is relatively soft. The possible explanation is that periodic addition of the metals may restrain the crystal growth of the nitrides. It is well known that decrease of crystallite size of a film may lead to the increase of the hardness [11]. This is one of the important reasons that the hardness of multilayer films was higher than that of monolayer films of the same total thickness in some cases. Moreover, ductility may be improved and residual stress may relax due to the addition of pure metals since the ductile metal layers between nitride layers may dissipate energy through plastic deformation, resulting in the increase of bonding strength [17].

Subramanian et al. [14] deposited TiN/Ti multilayer film of 200 layers with the total thickness of $1.5 \mu \mathrm{m}$ by reactive DC magnetron sputtering. The measured hardness was $13.73 \mathrm{GPa}$ for the TiN monolayer film and $25.52 \mathrm{GPa}$ for the TiN/Ti multilayer film. Abadias et al. [15] produced TiN/W multilayer films at room temperature by using ion beam sputtering. An increase in hardness was observed with decreasing modulation period, and the maximum value was 24.2 GPa. High bonding strength of TiN/W multilayer films was also reported in their paper [15].

Bulk tantalum has excellent physical and chemical properties such as good ductility, high melting point $\left(2996^{\circ} \mathrm{C}\right)$, and relatively low thermal conductivity $\left(57 \mathrm{~W} / \mathrm{m} \cdot \mathrm{K}\right.$ at $\left.20^{\circ} \mathrm{C}\right)$ 


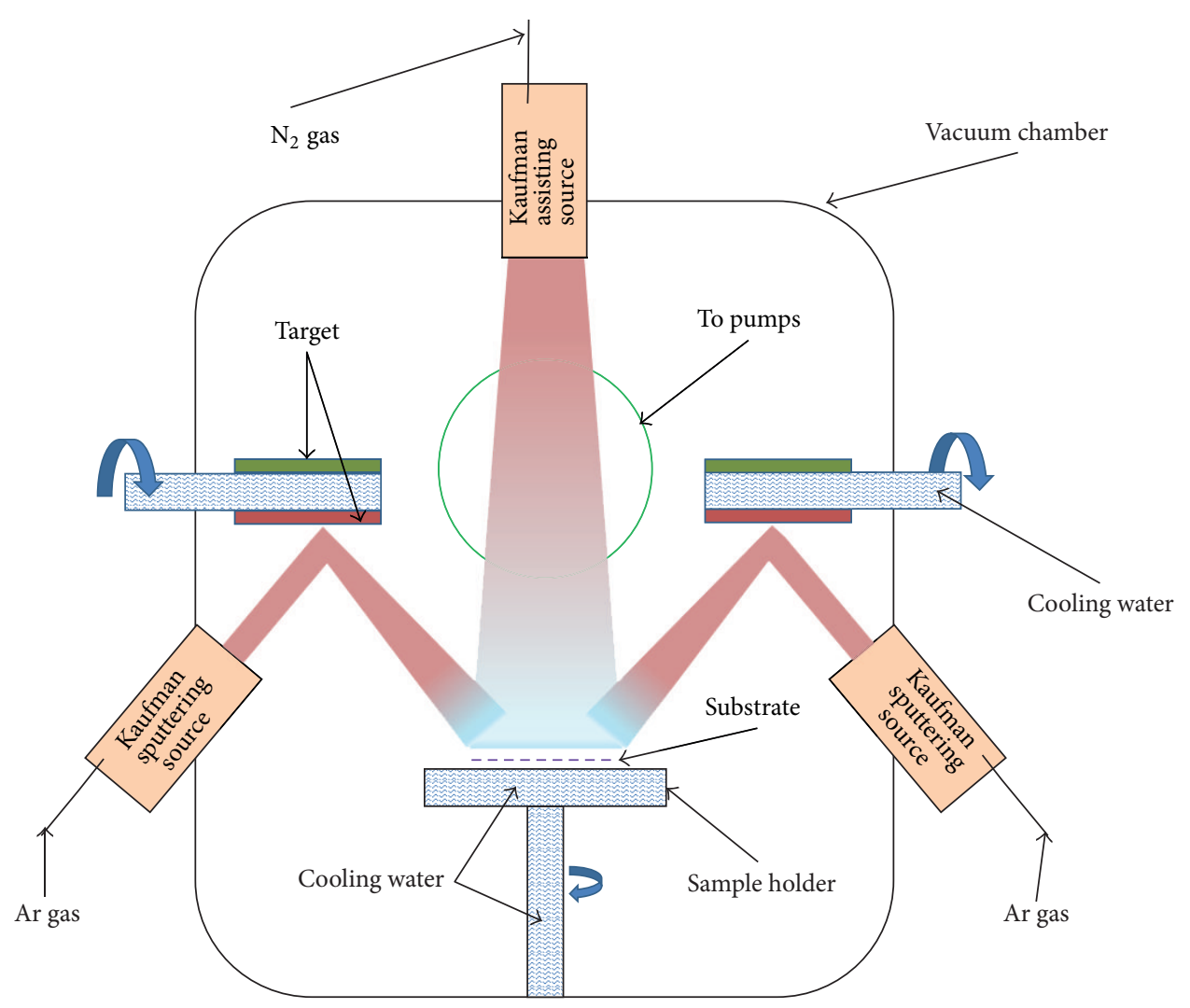

FIGURE 1: Schematic of the ion beam assisted deposition system.

[18]. Ta films deposited by physical vapor deposition have been used to protect large caliber military gun tubes [1822]. Kang et al. produced $\mathrm{Ta} / \mathrm{TaN}$ multilayer films on a high speed steel substrate by reactive sputtering and achieved a microvickers hardness of $2700 \mathrm{kgf} \mathrm{mm}^{-2}$ [23]. They pointed out that the interlocking between $\mathrm{Ta}$ and $\mathrm{TaN}$ in the interface became stronger as the modulation period decreased, and the bonding strength between them increased.

In the present work, TiN/Ta multilayer film was produced by introducing $\mathrm{Ta}$ sublayer to TiN film. Microstructure and mechanical properties of TiN/Ta multilayer film were investigated.

\section{Experimental}

Ion beam assisted deposition is an effective technique to prepare nanoscale films because the deposition parameters, such as beam current, beam energy, and ion flux, can be easily controlled. In the present study, an ion beam assisted deposition system was used to deposit TiN/Ta multilayer film. The schematic diagram of the system is shown in Figure 1. The system consists of three Kaufman ion sources, including two sputtering ion sources and one assisting ion source. It also consists of one sample holder and two target holders, all of them are rotatable and water-cooled. Each target holder has four working positions and four rectangular plates of targets could be installed. During deposition, the target holders are manually rotated to allow the required targets to be exposed to the sputtering $\mathrm{Ar}^{+}$beam to obtain desirable sublayer materials. In the present experiment, plates $(120 \mathrm{~mm} \times 100 \mathrm{~mm} \times 8 \mathrm{~mm})$ of $\mathrm{Ti}(99.95 \%)$ and $\mathrm{Ta}(99.95 \%)$ were used as the target materials.

One side polished silicon (100) wafers measuring $20 \mathrm{~mm}$ $\times 20 \mathrm{~mm} \times 0.5 \mathrm{~mm}$ were used as the substrate. Before deposition, the silicon wafers were ultrasonically cleaned in acetone and alcohol, then rinsed in deionized water each for 10 minutes and dried by hair dryer. In order to remove surface adsorbent and to activate surface atoms, the silicon (100) wafers were bombarded prior to deposition for 20 minutes with $\mathrm{Ar}^{+}$beam from the assisting ion source (the base pressure was $5.0 \times 10^{-4} \mathrm{~Pa}$, the working pressure was $5.0 \times$ $10^{-3} \mathrm{~Pa}$, beam energy $800 \mathrm{eV}$, beam current $60 \mathrm{~mA}$, and ion flux $9.6 \times 10^{14} \mathrm{ions} / \mathrm{cm}^{2} \cdot \mathrm{s}$ ).

A Ti transition layer was firstly deposited by sputtering Ti target with the $\mathrm{Ar}^{+}$beam for 10 minutes. Then the TiN/Ta multilayer film was deposited by alternately sputtering $\mathrm{Ti}$ and $\mathrm{Ta}$ with the $\mathrm{Ar}^{+}$beam from the sputtering ion source; the assisting $\mathrm{N}^{+}$beam was used to bombard the as-deposited $\mathrm{Ti}$ films to obtain the TiN sublayers. Depositions were made at room temperature. The base pressure was $4.0 \times 10^{-4} \mathrm{~Pa}$ and the working pressure was about $1.0 \times 10^{-2} \mathrm{~Pa}$. The detailed deposition parameters are listed in Table 1. A TiN monolayer film was also deposited for comparison. Deposition parameters of the TiN monolayer film are as follows: Ti target was sputtered by $\mathrm{Ar}^{+}$beam from the sputtering ion sources for $220 \mathrm{~min}$ with the beam energy of $2.5 \mathrm{keV}$, beam current of 
TABLE 1: Deposition parameters for TiN/Ta multilayer film.

\begin{tabular}{|c|c|c|c|c|}
\hline Layers & The sputtering ion source & The assisting ion source & $\begin{array}{l}\text { Deposition } \\
\text { time for } \\
\text { each layer }\end{array}$ & Number of layers \\
\hline Ti transition layer & $\begin{array}{c}\mathrm{Ar}^{+} \text {beam, beam energy } 2.5 \mathrm{keV} \text {, beam } \\
\text { current } 80 \mathrm{~mA} \text {, ion flux } \\
2.4 \times 10^{14} \text { ions } / \mathrm{cm}^{2} \cdot \mathrm{s}\end{array}$ & - & $10 \mathrm{~min}$ & 1 \\
\hline TiN sublayer & $\begin{array}{c}\mathrm{Ar}^{+} \text {beam, beam energy } 2.5 \mathrm{keV} \text {, beam } \\
\text { current } 80 \mathrm{~mA} \text {, ion flux } \\
2.4 \times 10^{14} \text { ions } / \mathrm{cm}^{2} \cdot \mathrm{s}\end{array}$ & $\begin{array}{c}\mathrm{N}^{+} \text {beam, beam energy } 0.2 \mathrm{keV} \text {, beam } \\
\text { current } 30 \mathrm{~mA} \text {, ion flux } \\
4.8 \times 10^{14} \text { ions } / \mathrm{cm}^{2} \cdot \mathrm{s}\end{array}$ & $1 \mathrm{~min}$ & 110 \\
\hline Ta sublayer & $\begin{array}{c}\mathrm{Ar}^{+} \text {beam, beam energy } 2.5 \mathrm{keV} \text {, beam } \\
\text { current } 80 \mathrm{~mA} \text {, ion flux } \\
2.3 \times 10^{14} \text { ions } / \mathrm{cm}^{2} \cdot \mathrm{s}\end{array}$ & - & $1 \mathrm{~min}$ & 110 \\
\hline TiN top layer & $\begin{array}{c}\mathrm{Ar}^{+} \text {beam, beam energy } 2.5 \mathrm{keV} \text {, beam } \\
\text { current } 80 \mathrm{~mA} \text {, ion flux } \\
2.4 \times 10^{14} \text { ions } / \mathrm{cm}^{2} \cdot \mathrm{s}\end{array}$ & $\begin{array}{c}\mathrm{N}^{+} \text {beam, beam energy } 0.2 \mathrm{keV} \text {, beam } \\
\text { current } 30 \mathrm{~mA} \text {, ion flux } \\
4.8 \times 10^{14} \text { ions } / \mathrm{cm}^{2} \cdot \mathrm{s}\end{array}$ & $1 \mathrm{~min}$ & 1 \\
\hline
\end{tabular}

$80 \mathrm{~mA}$, and ion flux of $2.4 \times 10^{14}$ ions $/ \mathrm{cm}^{2} \cdot \mathrm{s}$. The deposited film was simultaneously bombarded by $\mathrm{N}^{+}$beam with the beam energy of $200 \mathrm{eV}$, beam current of $30 \mathrm{~mA}$, and ion flux of $4.8 \times 10^{14}$ ions $/ \mathrm{cm}^{2} \cdot \mathrm{s}$, from the assisting ion source.

A field-emission transmission electron microscope (JEOL JEM2010F) equipped with an Energy Dispersive $\mathrm{X}$-ray spectroscopy system was used to investigate the cross-sectional structure of the TiN/Ta multilayer film. $\mathrm{X}$-ray diffraction analysis of the TiN/Ta multilayer film was performed using a D/max $2550 \mathrm{HB}+/ \mathrm{PC}$ X-ray diffractometer (Rigaku Co., Tokyo, Japan) in a grazing incidence X-ray scan using $\mathrm{Cu} \mathrm{K} \alpha$ radiation with a wavelength of $0.15418 \mathrm{~nm}$. Elemental composition-depth profiles of the $\mathrm{TiN} / \mathrm{Ta}$ multilayer film were constructed using a PHI-P700 scanning auger microscopy system. An electron-beam with the accelerating voltage of $5 \mathrm{kV}$ was used to excite auger electron emission from the sample surface. The ion incidence angle was about $30^{\circ}$ with respect to the sample surface. Thermal oxidation $\mathrm{SiO}_{2} / \mathrm{Si}$ was used as the reference material and the etching rate was $1 \mathrm{~nm} / \mathrm{min}$. Residual stress of the films was studied using a substrate curvature method, in which the radius of the curvature of the film/substrate was measured and the Stoney Equation was used to calculate the residual stress of the films. Curvature radius of the film/substrate system was measured by an ADE Phase Shift MicroXAM3D surface profilometer. Nanohardness $(H)$ and elastic modulus $(E)$ of the as-deposited TiN/Ta multilayer film were analyzed by a $\mathrm{NHT}^{2}$ nanoindentation tester (CSM Instruments SA). In all the tests, the measurement results of 3 indentations on different surface positions with a spacing of $20 \mu \mathrm{m}$ were averaged as the mean values. In order to study fracture toughness of the TiN/Ta multilayer film and the TiN monolayer film, a Tukon 2500 hardness testing machine with a diamond Vickers indenter from Wilson Hardness Group was used. The load of $0.2 \mathrm{~N}$ was applied. The testing dwell time was $10 \mathrm{~s}$. Bonding strength of the film/substrate was analyzed by a NST nanoscratch tester (CSM Instruments SA). Differential scanning calorimetric (DSC) and thermogravimetric analysis (TGA) (Netzsch STA 449F3) were performed in ambient air to study the thermal

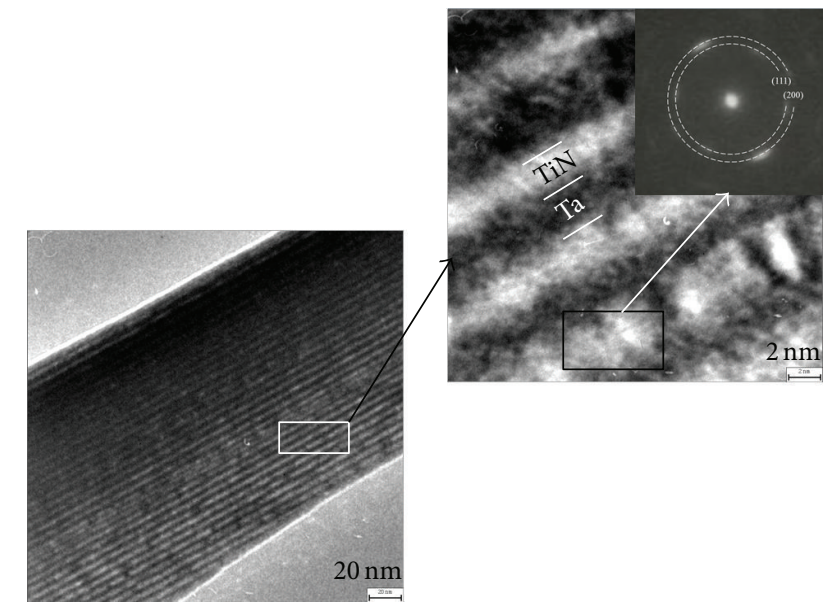

FIGURE 2: Cross-sectional bright field transmission electron micrograph and selected area electron diffraction pattern of the TiN/Ta multilayer film.

stability of the films. The heating rate was $10^{\circ} \mathrm{C} / \mathrm{min}$ and the temperature ranged from room temperature to $700^{\circ} \mathrm{C}$.

\section{Results and Discussion}

3.1. Microstructure. The as-deposited TiN/Ta multilayer film was observed by transmission electron microscope operated at $200 \mathrm{kV}$. The cross-sectional microstructure is shown in Figure 2. The modulation period and the individual layer thickness can be determined. The modulation period of the TiN/Ta multilayer film is $\sim 5.6 \mathrm{~nm}$. Both the TiN (bright) and Ta (dark) sublayers have the thicknesses of around $2.8 \mathrm{~nm}$. The selected area electron diffraction pattern of the TiN/Ta multilayer film shows a spotty-discontinuous ring pattern (see Figure 2). Diffraction ring matches with (111) plane of face centered cubic TiN phase. X-ray diffraction pattern (Figure 3) also shows the TiN (111) peak. As shown in Figure 3, the peak intensity of the TiN (111) orientation is the strongest one in both the TiN/Ta multilayer film and the 


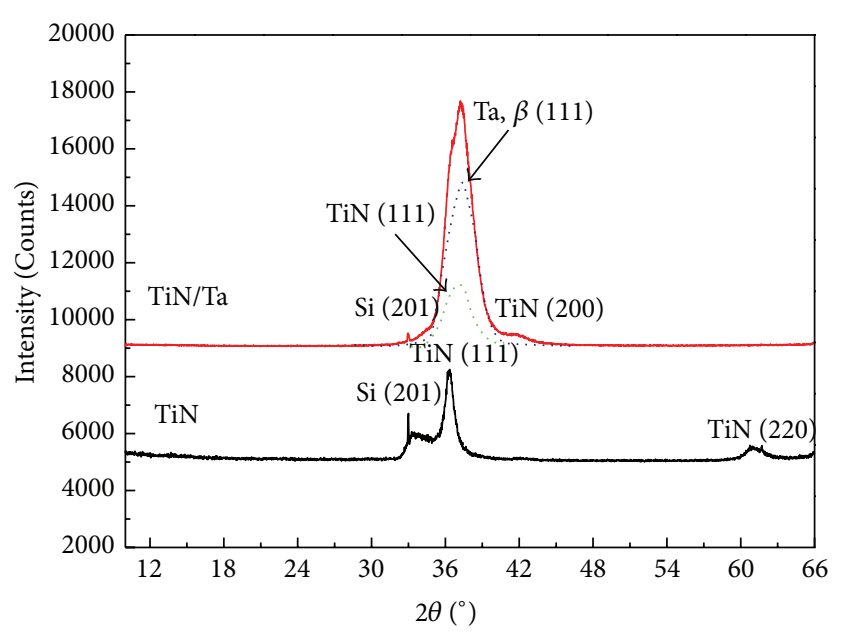

FIgURE 3: X-Ray diffraction patterns of the TiN/Ta multilayer film and the TiN monolayer film.

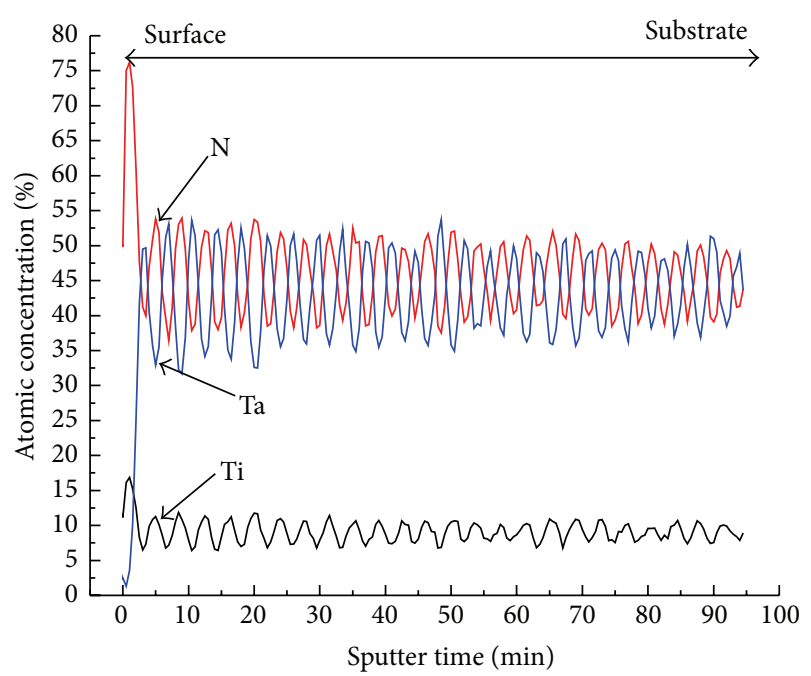

FIGURE 4: Auger electron spectroscopy depth profile of Ti, N, and Ta elements in the TiN/Ta multilayer film.

TiN monolayer film. From Figure 3, it can be seen that the TiN (111) peak in the TiN/Ta multilayer film is wider than in the TiN monolayer film, indicating that the TiN/Ta multilayer film has a much smaller grain size. The reason is that the Ta sublayers restrained the crystal growth of TiN, led to the decrease of the grain size. The broadening of the diffraction peaks in this case is possibly due to the nanocrystalline structure of the Ta film.

3.2. Chemical Composition. Figure 4 shows the Auger electron spectroscopy depth profile of the TiN/Ta multilayer film. A periodic variation of $\mathrm{Ti}, \mathrm{N}$, and $\mathrm{Ta}$ concentrations along film thickness is clearly seen. The thickness of the sublayers calculated from Figure 4 is about $2 \mathrm{~nm}$, which is not consistent with the result of TEM observation shown in Figure 2. The reason of the difference is that the etch rates of
TABLE 2: Calculation of residual stresses.

\begin{tabular}{lcc}
\hline & $\mathrm{TiN} / \mathrm{Ta}$ & $\mathrm{TiN}$ \\
\hline$R_{c}$ & $2593 \mathrm{~mm}$ & $1871 \mathrm{~mm}$ \\
$\sigma$ & $-4.8 \mathrm{GPa}$ & $-6.7 \mathrm{GPa}$ \\
\hline
\end{tabular}

the TiN/Ta multilayer film and thermal oxidation $\mathrm{SiO}_{2} / \mathrm{Si}$ are different.

3.3. Mechanical Properties. Physical vapor deposition methods usually generate large residual stresses inside films [24]. Residual stress of the TiN/Ta multilayer film and TiN monolayer film was studied using a substrate curvature method; the Stoney Equation [25] listed as follows was used to calculate the residual stress of the films:

$$
\sigma=\frac{E_{s}}{6\left(1-v_{s}\right)} \frac{t_{s}^{2}}{t_{f}}\left(\frac{1}{R_{c}}-\frac{1}{R_{\mathrm{uc}}}\right)
$$

where $\sigma$ is the residual stress, $E_{s}$ is the Young's modulus of substrate, $v_{s}$ is the Poisson ratio of substrate, $R_{\mathrm{uc}}$ is the radius of the curvature of the uncoated substrate, $R_{c}$ the radius of curvature of the coated substrate, and $t_{s}$ and $t_{f}$ are the thickness of the substrate and the film, respectively. In this paper, $E_{s}$ is $130 \mathrm{GPa}, v_{s}$ is $0.278, t_{s}$ is $0.5 \mathrm{~mm}, t_{f}$ is $0.6 \mu \mathrm{m}, R_{\mathrm{uc}}$ is $\infty$, and $R_{c}$ was measured.

Table 2 shows the measured curvature values and the calculated residual stress. The residual stresses of both the TiN/Ta multilayer film and the TiN monolayer film are compressive. An obvious reduction of the residual stress can be clearly seen for the multilayer. The residual stress of the TiN/Ta multilayer film is about $72 \%$ of that of the TiN monolayer film. It is suggested that the reduction of that of the residual stress was due to the introduction of the Ta sublayers, through which partial residual stress was released during deposition.

Figure 5 shows the loading and unloading curves in nanohardness tests for the TiN/Ta multilayer and the TiN monolayer films, in which an indentation load of $10 \mathrm{mN}$ was applied. Both nanohardness $(H)$ and elastic modulus $(E)$ of the TiN/Ta multilayer film and the TiN monolayer film were calculated from the plots of Figure 5. The results are plotted in Figure 6. It can be seen that the nanohardness of the TiN/Ta multilayer film is $24 \mathrm{GPa}, 14 \%$ higher than that of the TiN monolayer film. This is because the TiN/Ta multilayer film has smaller grain size than that of the TiN monolayer film.

The ratio $H^{3} / E^{2}$ can be used to measure the elasticity of a film. Particularly, a high value of $H^{3} / E^{2}$ is an indicator of high elasticity of the film [26]. The $H^{3} / E^{2}$ value of the TiN/Ta multilayer film, calculated from Figure 6, is about 0.28, 3.5 times as high as that of the TiN monolayer film. It means that the TiN/Ta multilayer film has higher elasticity than the TiN monolayer film.

Fracture toughness is one of the most important mechanical properties for thin solid films. Indentation test with a diamond Vickers indenter was performed to study the fracture toughness of the TiN/Ta multilayer film and the TiN monolayer film. Figure 7 presents the optical images 


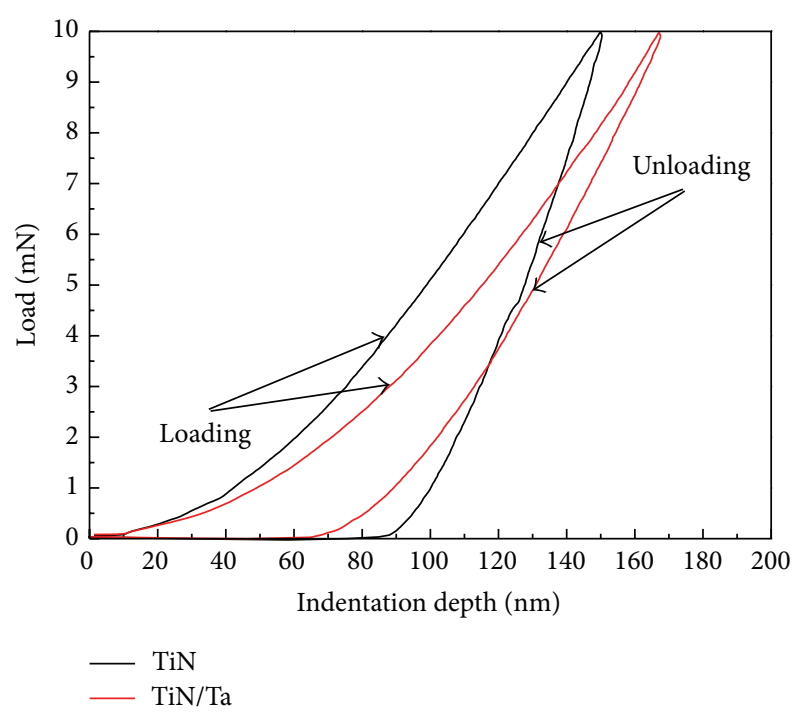

FIGURE 5: Loading and unloading curves for the TiN/Ta multilayer film and the TiN monolayer film.

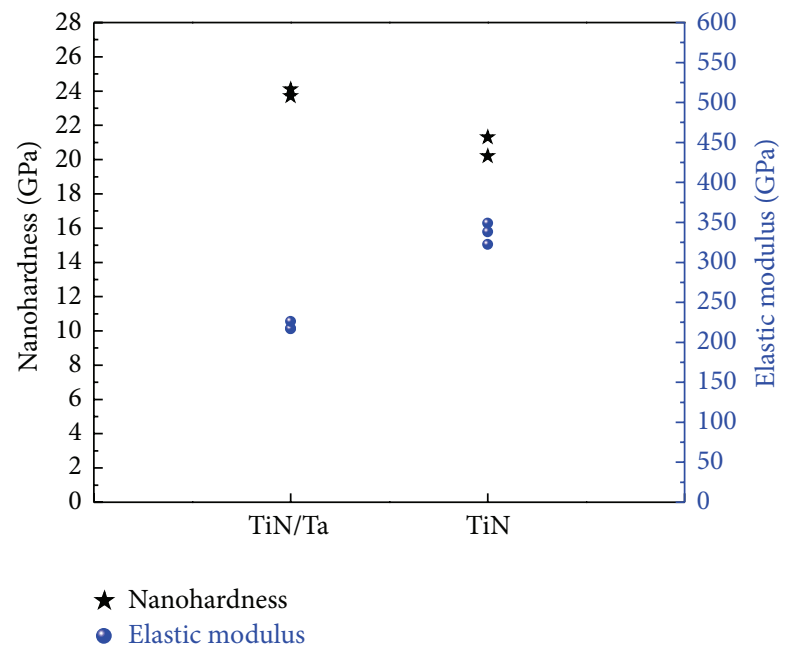

Figure 6: Nanohardness and elastic modulus of the TiN/Ta multilayer film and the TiN monolayer film.

of the indentation. Around the indentation area, significant delamination occurred for the TiN monolayer film. However, there is no obvious crack for the TiN/Ta multilayer film, suggesting that the TiN/Ta multilayer film has higher fracture toughness than the TiN monolayer film.

Theoretical analysis and experimental studies indicated that resistance to plastic deformation, fracture toughness, and wear resistance of thin solid films not only correlates with hardness $(H)$ and elastic modulus $(E)$ but also relates to the ratio $H^{3} / E^{2}$ [27-32]. The results of the present study showed that high value of $H^{3} / E^{2}$ corresponds to the high cracking resistance, which is consistent with the previous work. Low residual stress is beneficial to higher bonding strength, too.

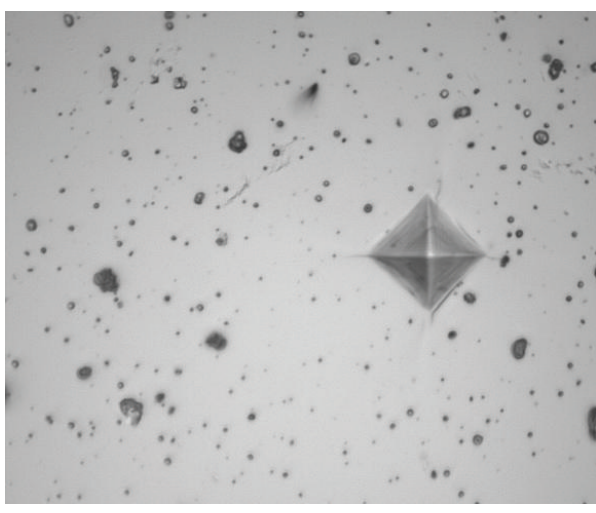

(a)

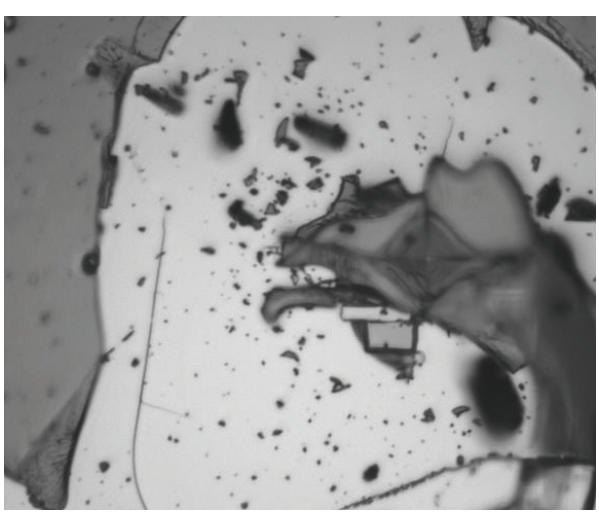

(b)

FIgUre 7: Optical images of the indentation (a) the TiN/Ta multilayer film and (b) the TiN monolayer film.

Bonding strength of a film to substrate is normally defined as the energy needed to separate the film from substrate. However, direct measurement of the energy remains difficult. Usually, nanoscratch test is applied to characterize the bonding strength. In a typical nanoscratch test, the critical load $L_{c}$, at which failure occurs, is used to evaluate the bonding strength of the film/substrate system. Figure 8 shows surface profiles and scratch tracks of the TiN/Ta multilayer film, as well as the TiN monolayer film. Surface profile of the TiN monolayer film indicates an abrupt increase point in residual depth. The normal load corresponding to this point is the critical load $L_{c}$. The $L_{c}$ of the TiN monolayer film is $45 \mathrm{mN}$ and that of the TiN/Ta multilayer film is around $75 \mathrm{mN}$. The scratch tracks also show a better bonding strength of the TiN/Ta film than the monolayer TiN film.

Ta has good ductility, it can dissipate energy through plastic deformation and increase the bonding between TiN and Ta. Hence, the cracking does not start at the TiN/Ta interface during the nanoscratch test. Moreover, the TiN/Ta multilayer film has higher elasticity and fracture toughness than the TiN monolayer film. All of them resulted in higher bonding strength of the TiN/Ta multilayer film.

3.4. Thermal Stability. DSC and TGA curves of the TiN/Ta multilayer film and the TiN monolayer film are shown in 


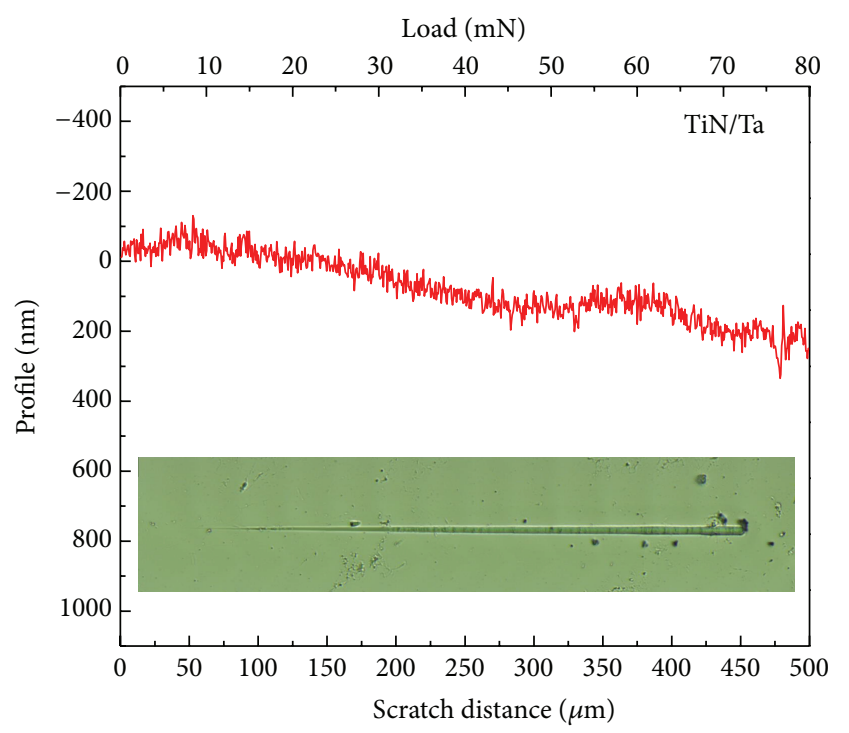

(a)

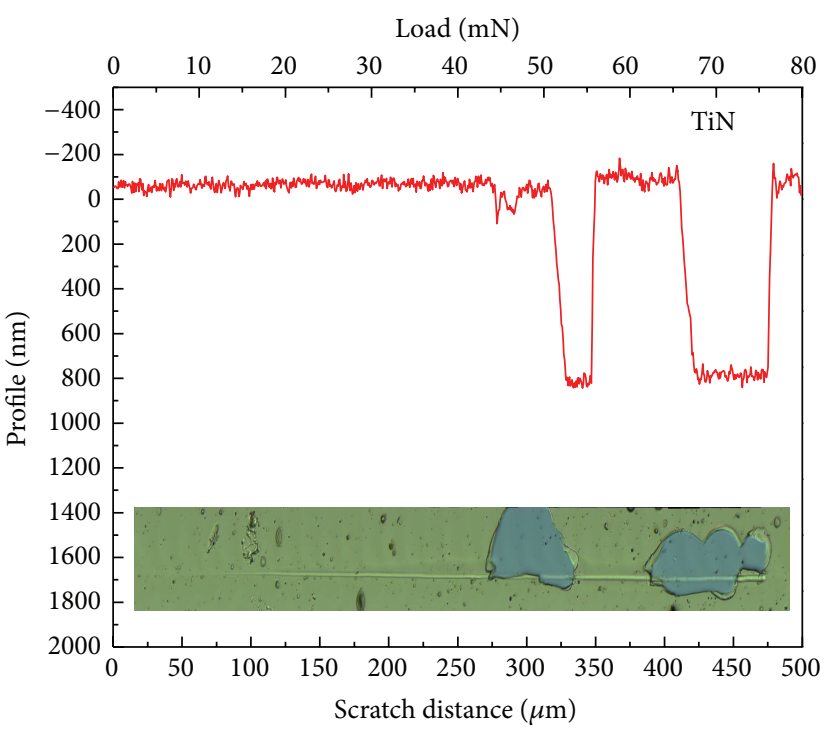

(b)

FIGURE 8: Surface profiles and scratch tracks on (a) the TiN/Ta multilayer film and (b) the TiN monolayer film.

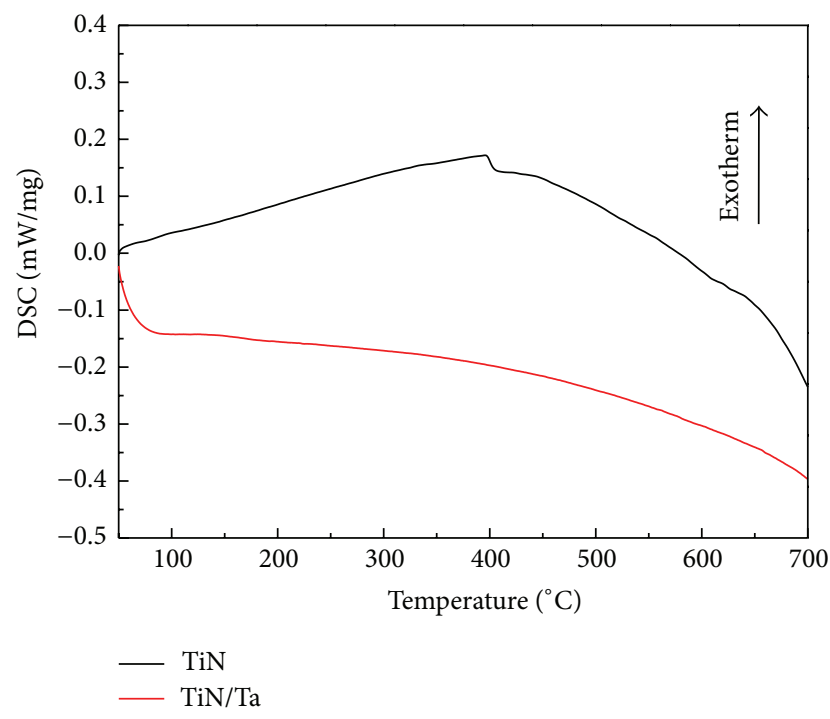

Figure 9: DSC curves of the TiN/Ta multilayer film and the TiN monolayer film.

Figures 9 and 10. For the TiN monolayer film, exothermic peak and weight loss due to oxidation were shown at about $400^{\circ} \mathrm{C}$. While for the TiN/Ta multilayer film, neither exothermic peak nor weight loss appeared during heating to $700^{\circ} \mathrm{C}$. Results of DSC and TGA indicate that the TiN/Ta multilayer film has better thermal stability than the TiN monolayer film.

\section{Conclusions}

TiN/Ta multilayer films with a modulation period of $5.6 \mathrm{~nm}$ and modulation ratio of $1: 1$ were prepared by ion beam assisted deposition. Compared to the monolayer TiN film

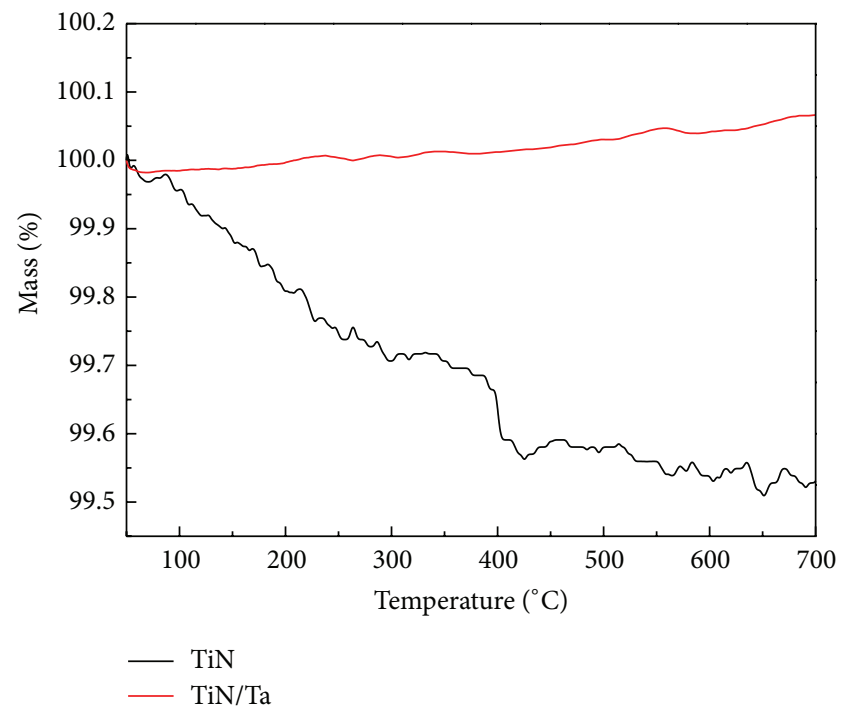

FIgure 10: TGA curves of the TiN/Ta multilayer film and the TiN monolayer film.

with the same thickness, an increase of nanohardness (14\%) was achieved for the TiN/Ta multilayer film. The increase of the nanohardness is due to the introduction of the $\mathrm{Ta}$ layers which restrained the growth of TiN crystal and led to the decrease of the grain size. Partial residual stress was released in the Ta sublayers during deposition, which led to the decrease of the residual stress of the TiN/Ta film. A significant increase of the $H^{3} / E^{2}$ value (3.5 times) indicated that the TiN/Ta multilayer film has higher elasticity than the TiN monolayer film. Attribute to the increase of elasticity and decrease of the residual stress, both the fracture toughness and the bonding strength of the TiN/Ta multilayer film 
obviously increased compared to that of the TiN monolayer film. Thermal stability of the TiN/Ta multilayer film was better than that of the TiN monolayer film.

\section{Conflict of Interests}

The authors declare that there is no conflict of interests regarding the publication of this paper.

\section{Acknowledgments}

The authors gratefully acknowledge the financial support from the National Natural Science Foundation of China (Grant no. 51075308/E0505) and the National Key Science and Technology Project (Grant no. 2010ZX04014-071).

\section{References}

[1] C. J. Tavares, L. Rebouta, B. Almeida et al., "Deposition and characterization of multilayered TiN/ZrN coatings," Thin Solid Films, vol. 317, no. 1-2, pp. 124-128, 1998.

[2] P. C. Yashar and W. D. Sproul, "Nanometer scale multilayered hard coatings," Vacuum, vol. 55, no. 3, pp. 179-190, 1999.

[3] W. D. Sproul, "New routes in the preparation of mechanically hard films," Science, vol. 273, no. 5277, pp. 889-892, 1996.

[4] H. C. Barshilia, A. Jain, and K. S. Rajam, "Structure, hardness and thermal stability of nanolayered TiN/CrN multilayer coatings," Vacuum, vol. 72, no. 3, pp. 241-248, 2003.

[5] K. J. Martin, A. Madan, D. Hoffman, J. Ji, and S. A. Barnett, "Mechanical properties and thermal stability of TiN/TiB ${ }_{2}$ nanolayered thin films," Journal of Vacuum Science \& Technology A, vol. 23, pp. 90-98, 2005.

[6] S. H. Yao, Y. L. Su, W. H. Kao, and T. H. Liu, "Tribology and oxidation behavior of TiN/AIN nano-multilayer films," Tribology International, vol. 39, no. 4, pp. 332-341, 2006.

[7] P. Sun, C. Su, T. Liou, C. Hsu, and C. Lin, "Mechanical behavior of TiN/CrN nano-multilayer thin film deposited by unbalanced magnetron sputter process," Journal of Alloys and Compounds, vol. 509, pp. 3197-3201, 2011.

[8] Y. H. Cheng, T. Browne, B. Heckerman, C. Bowman, V. Gorokhovsky, and E. I. Meletis, "Mechanical and tribological properties of TiN/Ti multilayer coating," Surface and Coatings Technology, vol. 205, no. 1, pp. 146-151, 2010.

[9] P.-L. Sun, C.-Y. Su, T.-P. Liou, C.-H. Hsu, and C.-K. Lin, "Mechanical behavior of TiN/CrN nano-multilayer thin film deposited by unbalanced magnetron sputter process," Journal of Alloys and Compounds, vol. 509, no. 6, pp. 3197-3201, 2011.

[10] S. H. Zhang, F. Cai, and M. X. Li, "The nanostructured phase transition and thermal stability of superhard $f$-TiN/h-AlSiN films," Surface and Coatings Technology, vol. 206, no. 17, pp. 3572-3579, 2012.

[11] N. Saoula, K. Henda, and R. Kesri, "Influence of Nitrogen Content on the Structural and Mechanical Properties of TiN Thin Films," Journal of Plasma and Fusion Research SERIES, vol. 8, pp. 1403-1407, 2009.

[12] H. F. Gong and T. M. Shao, "Influence of toughness on tribological performance of TiN/Ti multilayer coatings," Journal of Materials Engineering, vol. 10, pp. 26-31, 2009.

[13] N. Saoula, S. Djerourou, K. Yahiaoui et al., "Study of the deposition of Ti/TiN multilayers by magnetron sputtering," Surface and Interface Analysis, vol. 42, no. 6-7, pp. 1176-1179, 2010.
[14] B. Subramanian, R. Ananthakumar, and M. Jayachandran, "Structural and tribological properties of DC reactive magnetron sputtered titanium/titanium nitride (Ti/TiN) multilayered coatings," Surface and Coatings Technology, vol. 205, no. 11, pp. 3485-3492, 2011.

[15] G. Abadias, Y. Y. Tse, A. Michel, C. Jaouen, and M. Jaouen, "Nanoscaled composite TiN/Cu multilayer thin films deposited by dual ion beam sputtering: growth and structural characterisation," Thin Solid Films, vol. 433, no. 1-2, pp. 166-173, 2003.

[16] S. H. Yao, Y. L. Su, W. H. Kao, and K. W. Cheng, "A wear-resistant coating-Oxidized graded multilayer TiN/W coating," Materials Letters, vol. 64, no. 1, pp. 99-101, 2010.

[17] D. F. Bahr, J. W. Hoehn, N. R. Moody, and W. W. Gerberich, "Adhesion and acoustic emission analysis of failures in nitride films with a metal interlayer," Acta Materialia, vol. 45, no. 12, pp. 5163-5175, 1997.

[18] D. W. Matson, E. D. McClanahan, S. L. Lee, and D. Windover, "Properties of thick sputtered Ta used for protective gun cobe coatings," Surface and Coatings Technology, vol. 146-147, pp. 344-350, 2001.

[19] J. R. White, "Summary Abstract: a physical vapor deposition technique for plating gun tubes," Journal of Vacuum Science \& Technology A, vol. 4, pp. 2855-2856, 1986.

[20] D. W. Matson, M. D. Merz, and E. D. McClannahan, "High rate sputter deposition of wear resistant tantalum coatings," Journal of Vacuum Science \& Technology A, vol. 10, pp. 1791-1796, 1992.

[21] D. W. Matson, E. D. McClanahan, J. P. Rice, S. L. Lee, and D. Windover, "Effect of sputtering parameters on Ta coatings for gun bore applications," Surface and Coatings Technology, vol. 133-134, pp. 411-416, 2000.

[22] S. M. Maeng, L. Axe, T. A. Tyson, L. Gladczuk, and M. Sosnowski, "Corrosion behavior of magnetron sputtered $\alpha$-Ta coatings on smooth and rough steel substrates," Surface and Coatings Technology, vol. 200, no. 20-21, pp. 5717-5724, 2006.

[23] Y. K. Kang, C. M. Lee, and J. Lee, "Effects of processing variables on the mechanical properties of Ta/TaN multilayer coatings," Materials Science and Engineering, vol. 75, no. 1, pp. 17-23, 2000.

[24] C. J. Shute and J. B. Cohen, "Determination of yielding and debonding in $\mathrm{Al}-\mathrm{Cu}$ thin films from residual stress measurements via diffraction," Journal of Materials Research, vol. 6, no. 5, pp. 950-956, 1991.

[25] G. G. Stoney, "The tension of metallic films deposited by electrolysis," Proceedings of the Royal Society A, vol. 82, pp. 172$175,1909$.

[26] C. A. Charitidis and S. Logothetidis, "Effects of normal load on nanotribological properties of sputtered carbon nitride films," Diamond and Related Materials, vol. 14, no. 1, pp. 98-108, 2005.

[27] J. Musil, "Hard and superhard nanocomposite coatings," Surface and Coatings Technology, vol. 125, no. 1-3, pp. 322-330, 2000.

[28] P. H. Mayrhofer, C. Mitterer, and J. Musil, "Structure-property relationships in single- and dual-phase nanocrystalline hard coatings," Surface and Coatings Technology, vol. 174-175, pp. 725-731, 2003.

[29] D. Galvan, Y. T. Pei, and J. T. M. De Hosson, "Deformation and failure mechanism of nano-composite coatings under nanoindentation," Surface and Coatings Technology, vol. 200, no. 24, pp. 6718-6726, 2006.

[30] S. M. Yang, Y. Y. Chang, D. Y. Lin, D. Y. Wang, and W. $\mathrm{Wu}$, "Mechanical and tribological properties of multilayered TiSiN/CrN coatings synthesized by a cathodic arc deposition process," Surface and Coatings Technology, vol. 202, no. 10, pp. 2176-2181, 2008. 
[31] A. Czyżniewski, W. Gulbiński, G. Radnóczi, M. Szerencsi, and M. Pancielejko, "Microstructure and mechanical properties of W-C:H coatings deposited by pulsed reactive magnetron sputtering," Surface and Coatings Technology, vol. 205, pp. 44714479, 2011.

[32] C. Aguzzoli, C. A. Figueroa, F. S. de Souza, A. Spinelli, and I. J. R. Baumvol, "Corrosion and nanomechanical properties of vanadium carbide thin film coatings of tool steel," Surface and Coatings Technology, vol. 206, no. 10, pp. 2725-2731, 2012. 

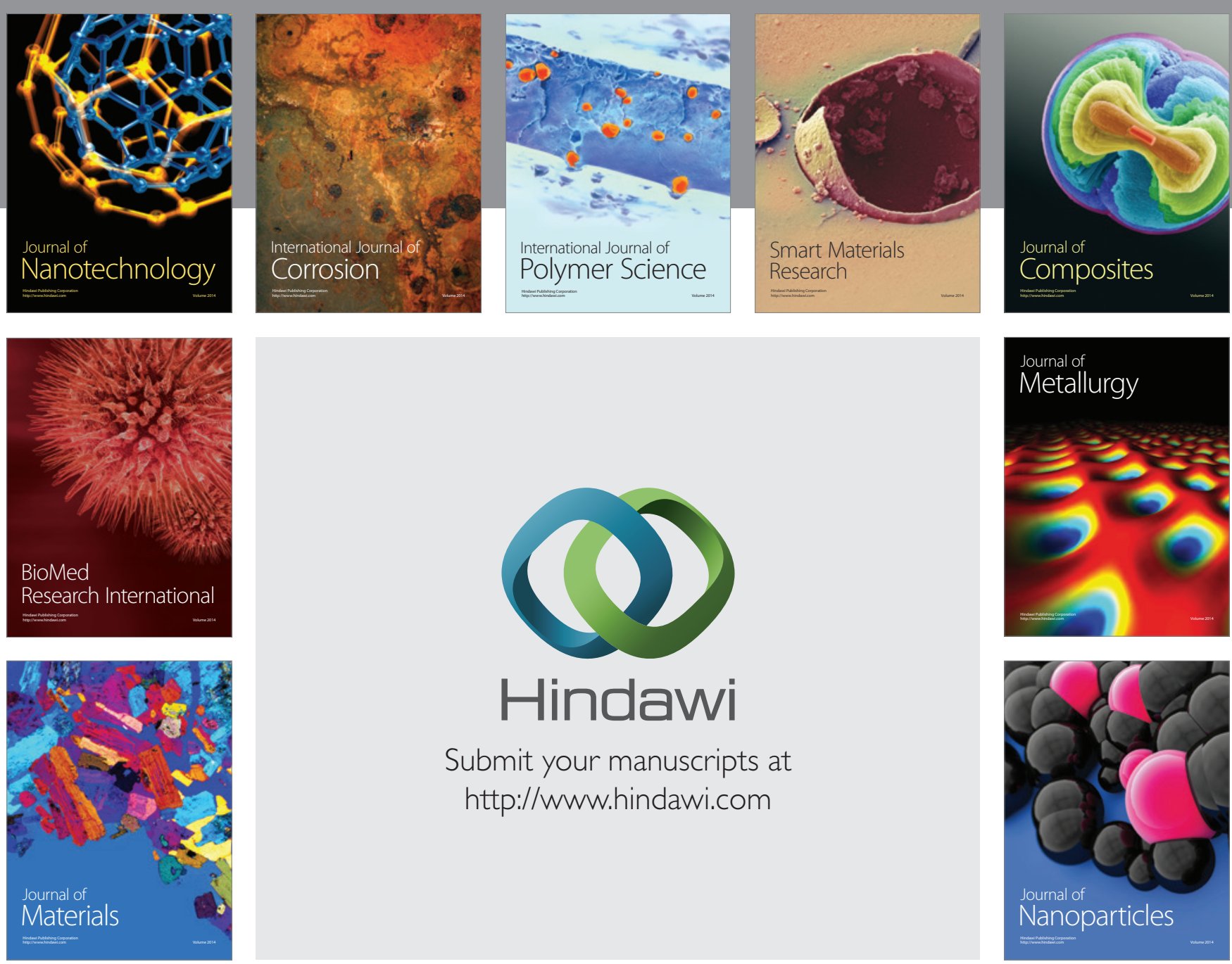

Submit your manuscripts at http://www.hindawi.com
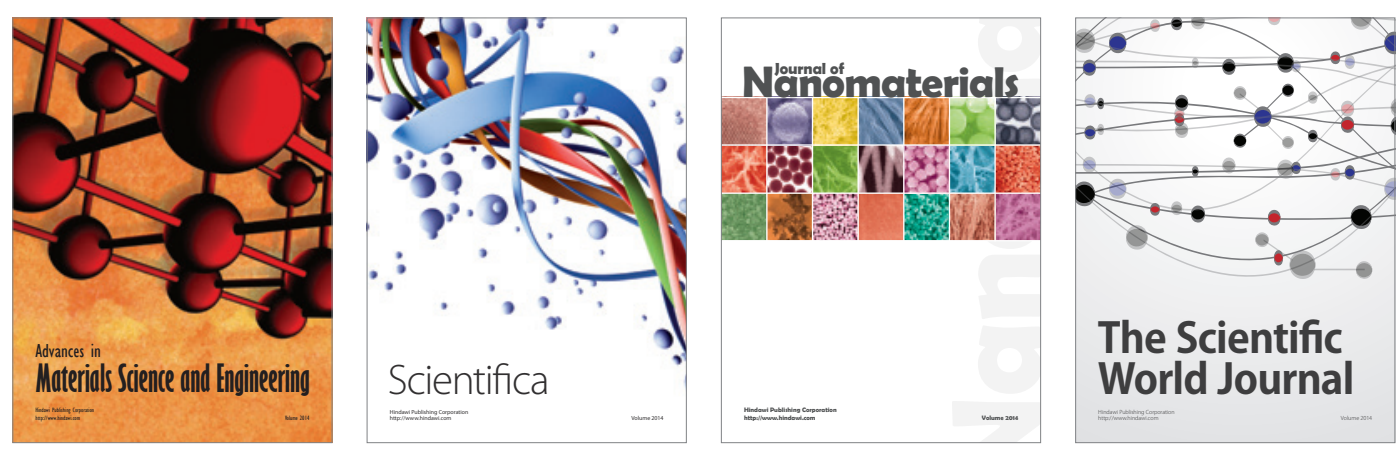

\section{The Scientific World Journal}
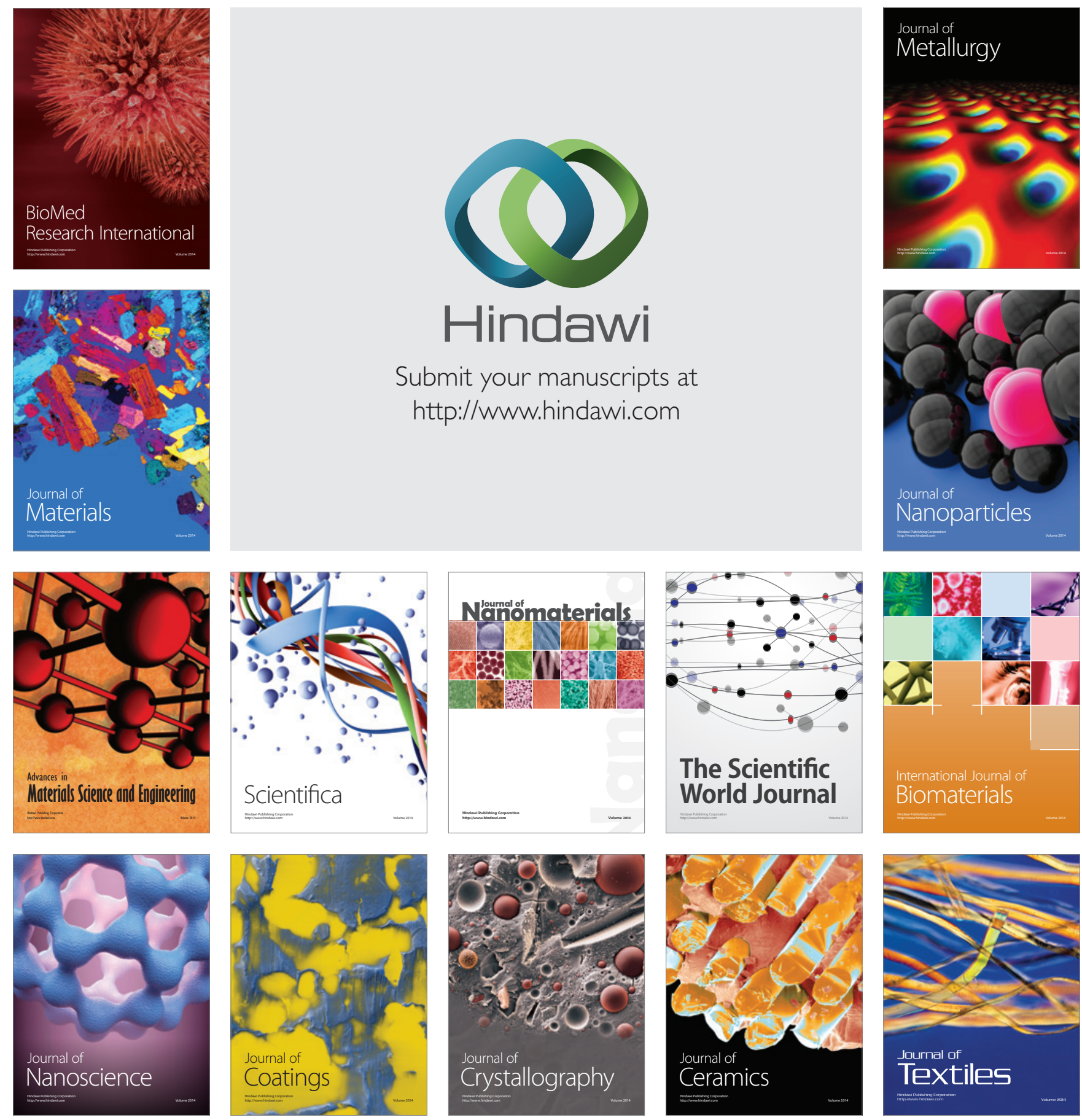